\title{
Stepwise Selection of Perceptual Texture Features
}

\author{
Antoni Grau, Joan Aranda and Joan Climent \\ Dept. of Computer Engineering and Automatic Control, Polytechnic University of Catalonia \\ e-mail: agrau@esaii.upc.es
}

\begin{abstract}
In computer vision, texture plays an important role. In this work we propose five human perceptual texture features heuristically extracted. Since a modeling can not be obtained from these features, we use a discriminant analysis technique to examine the discriminant power of each texture descriptor in order to select the most relevant. This task is done by a stepwise inclusion of variables indicating, furthermore, whether all of them are valuable and necessary producing a set of optimal discriminant variables. These selection procedures have been tested using Brodatz texture images, a benchmark in texture analysis.
\end{abstract}

Keywords. Texture analysis, texture features extraction, discriminant analysis, stepwise selection, Brodatz's album.

\section{Introduction}

The objective of texture segmentation is to separate an image into regions of distinct behavior under certain operators. An implicit assumption in this process is that the operators computation of each region is similar and that each region extends over a significant area. Therefore, it is reasonable to assume that image pixels which are spatially close are likely to be of the same texture. For this reason, the first task is to extract texture features which most completely embody information about the spatial distribution of texture in the image. In the past, a number of feature extraction methods have been proposed by different authors [VAN85]. A set of texture features is defined from a coocurrence matrix by [HAR73] and by [CON84]. Some authors find texture features based on others properties, such as Fourier transforms [MCL93], texture energy transforms [HSI89], fractal dimension [CHA95], among others. [TAM78] extracts features corresponding to human visual perception. The objective of this work is to present five texture features corresponding also to human visual perception, [GRA97], and to proof their necessity in segmenting textured images. To perform this proof, we will analyze the discriminant power of each feature in terms of discriminant analysis.

Feature selection plays a very important role in determining the overall performance of the system in the time and accuracy scale. Over the last decade multivariate data analysis techniques have seen wider acceptance and use in almost all fields of scientific inquiry. There are many reasons for this development, two of the more important are i) the realization that in many scientific inquiries it is necessary to analyze the simultaneous relationships among three or more variables; ii) the advent of the high speed computer with large storage capacity and the development of readily available and easy-to-use software packages for implementing multivariate analyses, [DIL84]. 
In following sections we will describe the texture feature extraction (section 2) and the resultant data set (section 3). In section 4 the feature selection algorithm will be rapidly shown. The results of this selection process can be seen in section 5 . The paper ends with the most relevant conclusions and future works.

\section{Problem Specification. Feature Extraction}

The proposed set of texture features have been heuristically selected based on the human perception of textures. The final goal (not described in this paper) of the whole image segmentation system which uses texture features is to work at high speed, that is, video rate. Due to this reason, the computed texture features are not very sophisticated. We search an admissible trade-off between their effectiveness in terms of quality and their low computational cost. For image segmentation using the texture information we propose a system that extracts texture features from the image and groups all the texture elements having something in common. There will be three different and separate steps to achieve the segmentation of the original image. In figure 1 the segmentation system is shown.

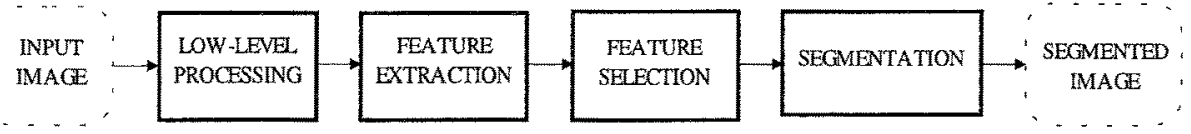

Fig. 1. Diagram of the image segmentation system using texture features.

In low-level processing the thinned edges of the image are obtained and the image is prepared for the feature extraction module. In this second module a feature space is generated. Each new point (represented as a vector) in this new space is a texel (texture element) which has texture descriptors as components. In the feature selection step, the degree of discriminating power will be evaluated in order to select the most relevant features. Then, the segmentation output is represented as the borders between texture groups.

The features extracted are mainly based on textural properties rather than structural ones because these provide a good set of tools for low-level segmentation in scene analysis. Being perceptible is the principal characteristic of the texture parameters and this perceptible nature of the parameters allow to compute their value with masks created in a perceptible manner too. These masks are local boolean expressions which are applied over each pixel in the image. Due to the masks, it will appear some constraints when the system will try to discriminate the textures, but in the other hand, there is a predisposition for a hardware implementation of the masks to find the texture parameters. The definitions of the parameters are as follows:

1. Straightness. This parameter indicates the straight line density over a region, and it has been derived from the linear regression model.

2. Blurriness. The blurriness is a visual effect where a progressive and slow gray level increasing or decreasing along an image area is noticed. We only consider blur 
pixels if the change in their intensity is inside a fixed interval, otherwise we consider sudden changes.

3. Abruptness. This parameter indicates sudden changes in the directionality of the texture.

4. Granularity has a high perceptual significance in textures. This value will indicate how many elements in the image are isolated or non-concatenated.

5. Discontinuity. This parameter measures the density of cut edges in the image. When the edges are continuous the value will be high.

Each texel determines a point in the 5-dimension texture space. The images used in this work contain 32-by-32 texels each and this will be the number of points in the feature space. The parameters can be found in a parallel manner because it does not exist any data dependence among them. Then, for an input image, where the minimum element is the pixel, each parameter is calculated in parallel with its masks to produce an output image where the minimum element is the texel (see figure 2). The size of the masks used to calculate the parameters is 4 -by-4 elements. A bigger size will generate a large amount of masks for each parameters and the cost in time will be excessive for our intentions. A smaller size of the masks is contradictory with the own definition of the parameters.

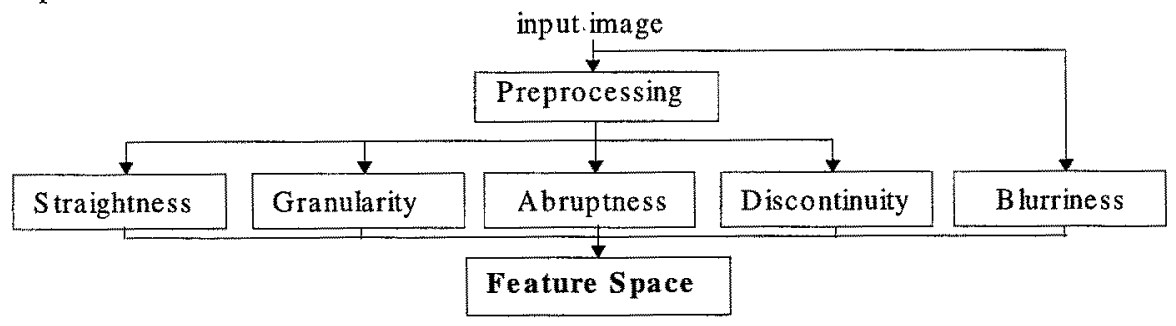

Fig. 2. Feature extraction and generation of the feature space.

The masks are calculated through the image following the raster scan. Therefore, every pixel in the image will be analyzed. These masks are template matching masks.

\section{Data Set}

In this work we take the images from Brodatz's album, [BRO66], which are used in many tests to evaluate texture analysis and algorithms. Also, in order to make a relevant texture test, we will treat 26 images. Each image is enough different from the others to create a wide variation of textures. In table 1 there are the name of each texture image and the assigned tag in the album. We assume that each image has the same degree of texture along the whole image. If this assumption was not completely true there is not any inconvenience whether some texels do not belong to the same texture, because it will be seen as a misclassification of those texels.

The data set is as follows: for each image we compute 32-by-32 texels with five components each, that is 1024 texels per image. Together with the components of the texels, there is the class to which these texels belong. 
The feature space element is $P=(\mathrm{S}, \mathrm{B}, \mathrm{A}, \mathrm{G}, \mathrm{D})$, where each component of the $5 \mathrm{D}$ point is the evaluated degree of texture, straightness, blurriness, abruptity, granularity and discontinuity, ranging their value from 0 to 255. Therefore, the feature space is made up with $P_{\mathrm{i}, \mathrm{j}}$ elements, where $i$ is the number of texels per image $(i=0, \ldots, 1023$ ) and $j$ is the class $(j=0, \ldots, 25)$, the class is the number of image. The size of the feature space is 26,624 points which have been normalized in a previous step.

\begin{tabular}{|c|c|c|c|c|c|}
\hline Texture & $\begin{array}{r}\text { image } \\
\text { tag }\end{array}$ & Texture & $\begin{array}{r}\text { image } \\
\text { tag }\end{array}$ & Texture & $\begin{array}{r}\text { image } \\
\text { tag }\end{array}$ \\
\hline Beach Sand & D28 & Marble & D59 & Grass & D9 \\
\hline Beach Sand & D29 & Mica & D5 & Ice & D100 \\
\hline Raffia & D84 & Paper & D109 & Beans & D74 \\
\hline Leather & D24 & Beach Pebbles & D54 & Brick wall & D94 \\
\hline Straw & D15 & Pig skin & D92 & Beach Pebbles & D23 \\
\hline Burlap & D103 & Sea fan & D87 & Barktree & D12 \\
\hline Reptile & $\mathrm{D} 22$ & Tree & D72 & Water & D38 \\
\hline Her, weave & D16 & Ricepaper & D108 & Pellets & D67 \\
\hline Cork & D32 & Fieldstone & D2 & & \\
\hline
\end{tabular}

Table 1. Texture images from [BRO66].

Theoretically, the texels evaluated for each image belong to the same class and they form an isolated cloud in the feature space. In practice, the texture images are not pure and it will appear some blends among textures. This effect will produce non-isolated groups and we will proof the degree of discrimination of each texture descriptor, that is, its power in separating each texture group.

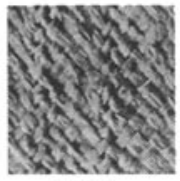

D84

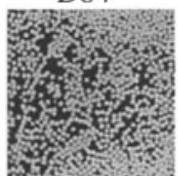

D67

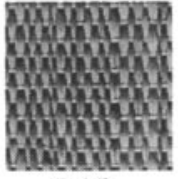

D15

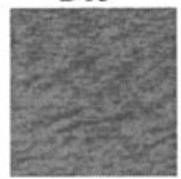

D38

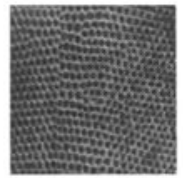

D22

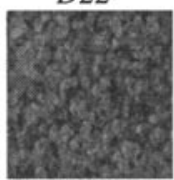

D29

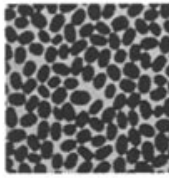

D74

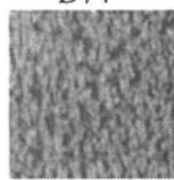

D24

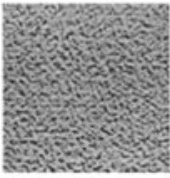

D109

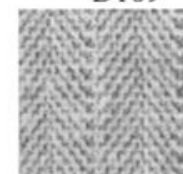

D16

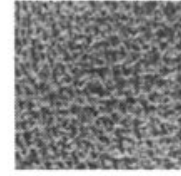

D92

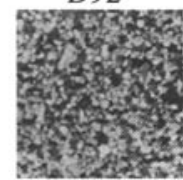

D54

Fig. 3. Texure images, from [BRO66].

In figure 3, some texture images are shown. The tag below each picture is the number in the Brodatz's album.

An assumption is that each group is drawn from a population which has a multivariate normal distribution. Such a distribution exists when each variable has a normal distribution about fixed values on all the others. This permits the precise computation of tests of significance and probabilities of group membership. If this assumption 
would be violated, the computed probabilities will be not exact, but they might still be quite useful if interpreted with caution. [LAC75] has shown that discriminant analysis is not particularly sensitive to minor violations of the normality assumption. The consequence is some reduction in efficiency and accuracy.

\section{Proposed Solution. Feature Selection.}

In concrete, our interest centers on the association between two sets of variables, where one set is the realization of a dependent measure. These methods are the dependence methods, and the technique proposed is the multiple discriminant analysis. This statistical technique is used for classifying individuals or objects into mutually exclusive and exhaustive groups in the basis of a set of independent variables.

Due to the acquisition of our data set, we do not know explicitly that the variables are good discriminators. We can not modeling mathematically each variable and to proof their discriminatory power. Although they may be good discriminators on their own, the redundant variables do not contribute to the analysis, thus it is advisable to eliminate weak or redundant variables. Their presence only complicates the analysis and they may even increase the number of misclassifications. The proposed solution to eliminate unnecessary variables is by using a stepwise procedure to select the most useful discriminating variables [KLE80]. This procedure involves forward and backward selection. In forward selection procedure each step starts with a review of the variable previously selected. If any of these variables no longer makes a sufficient contribution to the discrimination, it is cast out although it remains eligible for reselection at a future step. The removal of previously selected variables is usually the result of shared discriminating information with other variables selected on intervening steps. At the time it was selected, this variable may have made a unique contribution. However, variables selected on subsequent steps may combine with one another or with variables selected earlier to duplicate the contribution of this variable. The variable is then redundant and a candidate for removal.

\subsection{Stepwise Inclusion of Variables.}

The selection criteria in stepwise procedures employs Wilks' lambda criterion. This statistic takes in consideration both the differences between groups and the cohesiveness or homogeneity within groups.

We will assume that samples of $n_{i}$ observations are available from the $i$ th group, $i=$ $1,2, \ldots, K$, with each observation consisting of $p$ measurements $\mathbf{X}^{\prime}=\left(\mathrm{X}_{1}, \mathrm{X}_{2}, \ldots, \mathrm{X}_{p}\right)$. We denote by $\mathbf{T}$ the matrix of the total mean corrected sum-of-squares of crossproducts for the scores on all $n=\Sigma_{i} n_{i}$ observations. That is,

$$
\mathbf{T}=\sum_{i=1}^{K} \sum_{j=1}^{n_{i}}\left(\mathbf{X}_{i j}-\bar{x}\right)\left(\mathbf{X}_{i j}-\bar{x}\right)^{\prime}
$$


The matrix of sums-of-squares and cross-products for the th group will be denoted by $\mathbf{W}_{\mathrm{i}:}$

$$
\mathrm{W}_{\mathrm{i}}=\sum_{j=1}^{n_{i}}\left(\mathrm{X}_{i j}-\bar{x}\right)\left(\mathrm{X}_{i j}-\bar{x}\right)^{\prime}
$$

The within-groups sums-of-squares are given by $\mathbf{W}=\mathbf{W}_{1}+\mathbf{W}_{2}+\ldots+\mathbf{W}_{\mathrm{k}}$ (3). The matrix of between-groups sums-of-squares and cross-products can thus be found by the difference $\mathbf{B}=\mathbf{T}-\mathbf{W}(4)$. Define the linear compound $\mathbf{Y}=\hat{\mathbf{b}}^{\prime} \mathbf{X}$, the caret is used to denote sample-based estimates. With respect to the linear composite $\mathbf{Y}$, the between-groups sums-of-squares are given by $\hat{\mathbf{b}}^{\prime} \mathbf{B} \hat{\mathbf{b}}$. Similarly, the within-groups sums-of-squares are $\hat{\mathbf{b}}^{\prime} \mathbf{W} \hat{\mathbf{b}}$. We can define by $\hat{\lambda}=\frac{\hat{\mathbf{b}}^{\prime} \mathbf{B} \hat{\mathbf{b}}}{\hat{\mathbf{b}}^{\prime} \mathbf{W} \hat{\mathbf{b}}}$ (5) the ratio of the between-groups to within groups sums-of-squares for the $K$ groups. We wish to maximize $\hat{\lambda}$ with respect to $\hat{\mathbf{b}}$. We take the partial derivative with respect to $\hat{\mathbf{b}}$ and set it equal to zero. After some simplification we have $\left(\mathbf{W}^{-1} \mathbf{B}-\hat{\lambda}\right) \hat{\mathbf{b}}=\mathbf{0}$ (6). The maximum value $\hat{\lambda}$ is the largest eigenvalue of the matrix $\mathbf{W}^{\mathbf{1}} \mathbf{B}$, where $\hat{\lambda}_{1}, \hat{\lambda}_{2}, \ldots, \hat{\lambda}_{r}$ are its eigenvalues; $\hat{\mathbf{b}}$ is the corresponding eigenvector, whose elements are the discriminant weights associated with the first linear composite.

If we define $\mu_{j}, \mathrm{j}=1,2, \ldots, K$, as the mean vector of each group, the hypothesis of general interest is $\mathrm{H}_{0}: \mu_{1}=\mu_{2}=\ldots=\mu_{K}$ and test it against the alternative that not all of the $\mu_{j}$ 's are equal. To test $\mathrm{H}_{0}$ against $\mathrm{H}_{1}$ the Wilks's lambda likelihood-ratio criterion is defined as $\Lambda=\frac{|\mathbf{W}|}{|\mathbf{W}+\mathbf{B}|}$ (7). Thus, if we use the eigenvalue form, $\Lambda=\prod_{j=1}^{r}\left(1+\hat{\lambda}_{j}\right)^{-1}$.

At each step the model is examined. If the variable in the model that contributes least to the discriminatory power of the model as measured by Wilks' lambda fails to meet the criterion to stay, then that variable is removed. Otherwise, the variable not in the model that contributes most to the discriminatory power of the model is entered. When all variables in the model meet the criterion to stay and none of the other variables meets the criterion to enter, the stepwise selection process stops.

\section{Results}

For our purposes, we will use the SAS program and the procedure is stepdisc called as follows:

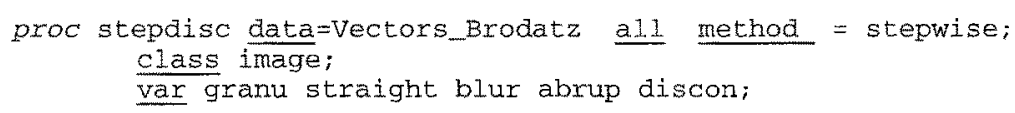

The output for the above procedure call is the next. 
Stepwise Discriminant Analysis
26624 Observations
26 class Levels
5 Variable(s) in the Analysis
0 Variable(s) will be included

The Method for Selecting Variables will be: STEPWISE

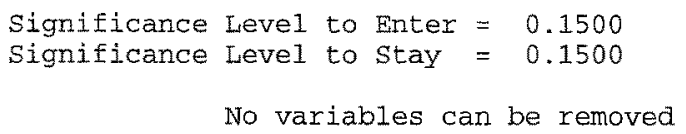

No further steps are possible

Stepwise Selection: Summary

\begin{tabular}{|c|c|c|c|c|c|}
\hline \multirow[b]{2}{*}{ Step } & Variable & \multirow{2}{*}{$\begin{array}{c}\text { Number } \\
\text { In }\end{array}$} & \multirow{2}{*}{$\begin{array}{r}\text { Partial } \\
\mathbb{R}^{* * 2}\end{array}$} & \multirow{2}{*}{$\begin{array}{c}F \\
\text { Statistic }\end{array}$} & \multirow{2}{*}{$\begin{array}{l}\text { Prob > } \\
\text { F }\end{array}$} \\
\hline & Entered Removed & & & & \\
\hline 1 & BEUR & 1 & 0.7701 & 3564.134 & 0.00 \\
\hline 2 & DISCON & 2 & 0.35 & 691. & 0.00 \\
\hline 3 & STRAIGHT & 3 & 0.3954 & 695.639 & 0.00 \\
\hline 4 & ABRUP & 4 & 0.1759 & 227.082 & 0.00 \\
\hline 5 & GRANU & 5 & 0.1843 & 240.423 & 0.000 \\
\hline
\end{tabular}

Stepwise selection: Summary

\begin{tabular}{|c|c|c|c|c|c|}
\hline \multirow[b]{2}{*}{ Step } & \multicolumn{2}{|c|}{ Variable } & \multirow{2}{*}{$\begin{array}{c}\text { Number } \\
\text { In }\end{array}$} & \multirow{2}{*}{$\begin{array}{l}\text { Wilks: } \\
\text { Lambda }\end{array}$} & \multirow{2}{*}{$\begin{array}{l}\text { Prob < } \\
\text { Lambda }\end{array}$} \\
\hline & Entered & Removed & & & \\
\hline 1 & BLUR & & 1 & 0.22988497 & 0.000 \\
\hline 2 & DISCON & & 2 & 0.1393 & 0.0 \\
\hline 3 & STRAIGHT & & 3 & 0.08422625 & 0.000 \\
\hline 4 & ABRUP & & 4 & 0.06940982 & 0.00 \\
\hline 5 & GRANU & & 5 & 0.05661428 & 0.0 \\
\hline
\end{tabular}

Three different procedures have been proved (forward, backward and stepwise), and the equal results have been found. The variables have been entered and none has been removed. Then, the null hypothesis $\mathrm{H}_{0}$ is rejected, and the mean vector of each texture group is different. From these results, we can observe that not all the variables cooperate in the same degree in the discrimination process. The most powerful descriptor is the blurriness, because it uses the gray level information instead the contour information used by the other descriptors.

This result can be interpreted as all variables give relevant information to obtain the groups from the input samples and the discrimination will be effective among different textures. In conclusion, the feature selection step has selected the five variables which will be used in our vision system.

\section{Conclusions}

In this work, we have proposed five perceptual texture features obtain in a heuristic manner. The use of a discriminant technique will help us to select the most valuable and useful variables. A stepwise selection of variable has been chosen. We have seen that the Wilks' lambda is a general statistic for handling tests of mean differences in multivariate data analysis settings. In fact, under certain conditions. such familiar 
statistics as $F$, Hotelling's $T^{2}$, and Mahalanobis' $D^{2}$ can all be viewed as special cases of $\Lambda$. However, the relatively simple transformations that convert $\Lambda$ exactly to these more familiar statistics no longer hold when multiple criteria and multiple groups are involved. The models selected by the used procedure are not necessarily the best possible models, and Wilks' lambda may not be the best measure of discrimination power but the in our application is not necessary to use others models because the overall probability of rejecting null hypothesis is much larger than the significance level.

As future work, we plan two strategies, first the addition of texture descriptors that use gray level information from the image but suitable for implementation with specific architecture, and second, structural features which are usually more problem-oriented can be added to the existing feature functions in order to tune the overall performance of the system to the specific problem.

\section{References}

[BRO66] Brodatz, P., Textures: A Photographic Album for Artists and Designers, Dover Publishing Co., New York, 1966.

[CHA95] Chaudhuri, B.B., and Sarkar, N., "Texture Segmentation Using Fractal Dimension", IEEE Trans. on Pattern Analysis and Machine Intelligence, Vol. PAMI17 No. 1, pp. 72-77, 1995.

[CON84] Conners, R.W., Trivedi, M.M. and Harlow, C.A., "Segmentation of a highresolution urban scene using texture operators", Comput. Vision Graphics and Image Proc., no. 25, pp. 273-310, 1984.

[DIL84] Dillon, W.R. and Goldstein, M., Multivariate Analysis. Methods and Applications, Wiley Series in Probability and Mathematical Statistics, 1984.

[GRA97] Grau, A., "Multiparametric Texture Feature Extraction Method oriented to Image Segmentation", Ph.D. Thesis, Polytechnic University of Catalonia, Barcelona, 1997.

[HAR73] Haralick, R.M., Shanmugam, K. and Dinstein, I. "Textural Features for Image Classification", IEEE Trans. on SMC, Vol. SMC-3, no. 6, pp. 610-621, 1973.

[HSI89] Hsiao, J.Y., and Sawchuk, A.A., "Supervised Textured Image Segmentation Using Feature Smoothing and Probabilistic Relaxation Techniques", IEEE Trans. on Pattern Analysis and Machine Intelligence, Vol. PAMI-11, pp. 1279-1291, 1989.

[KLE80] Klecka, W.R., Discriminant Analysis, Sage University Paper Series on Quantitative Applications in the Social Sciences, Series No. 07-19, Beverly Hills: Sage Publications, 1980.

[LAC75] Lachenbruch, P. A., Discriminant Analysis. New York: Hafner. 1975.

[MCL93] McLean, G.F., "Vector Quantization for Texture Classification", IEEE Trans. on Systems, Man and Cybernetics, Vol. SMC-23, No. 3, pp. 637-649, 1993.

[TAM78] Tamura, H., Mori, S., and Yamawaki, T., "Textural Features Corresponding to Visual Perception", IEEE Trans. on Systems, Man and Cybernetics, Vol. SMC-8, No. 6, pp. 460-473, 1978.

[VAN85] Van Gool, L., Dewaele P. and Oosterlinck, A, "Survey- texture analysis anno 1983", Comput. Vision Graphics and Image Processing, no. 29, pp. 336-357, 1985 . 